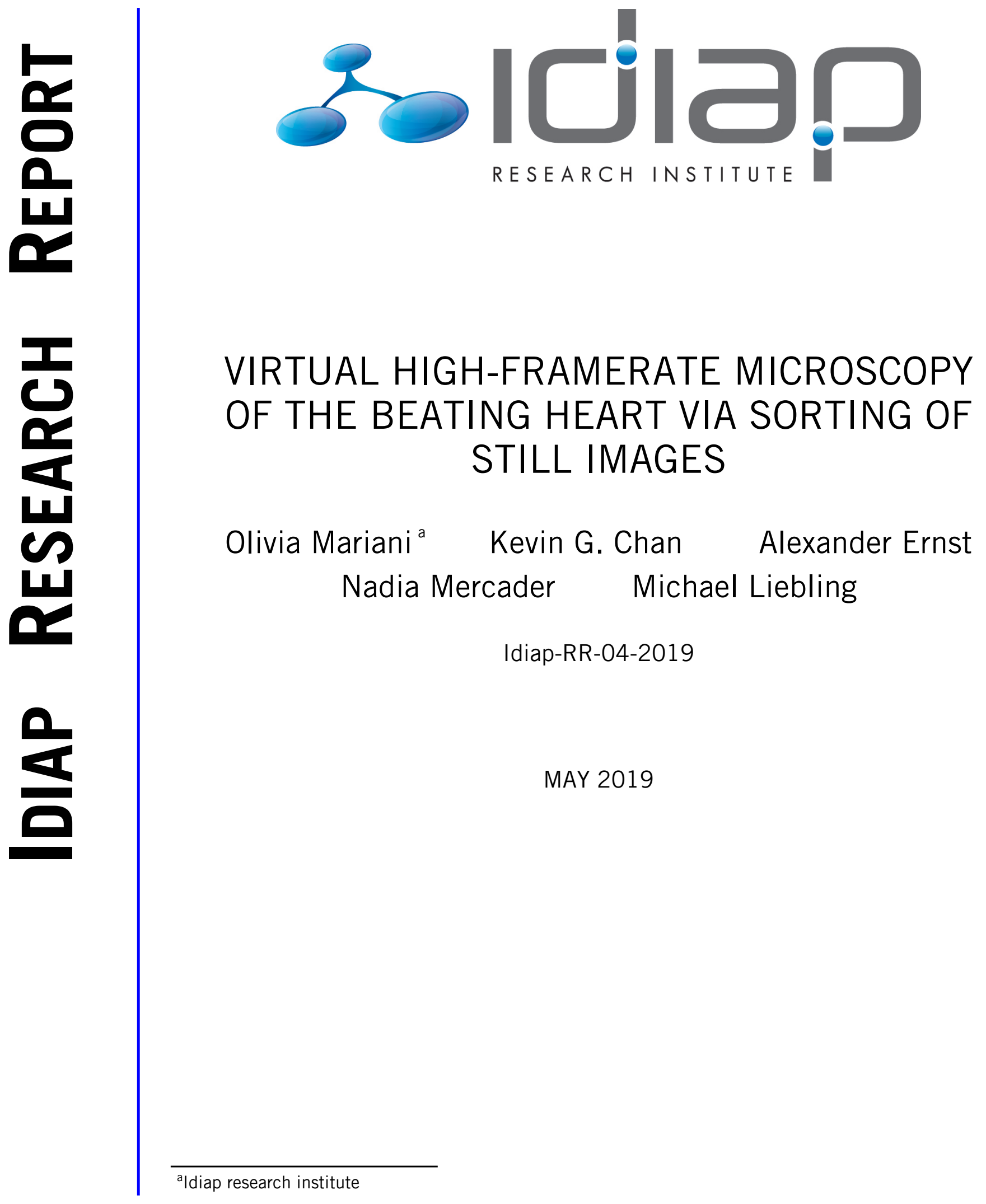





\title{
VIRTUAL HIGH-FRAMERATE MICROSCOPY OF THE BEATING HEART VIA SORTING OF STILL IMAGES
}

\author{
Olivia Mariani ${ }^{1,2}$, Kevin $_{\text {Chan }}^{3}$, Alexander Ernst ${ }^{4}$, Nadia Mercader $^{4}$, Michael Liebling $^{1,3}$ \\ ${ }^{1}$ Idiap Research Institute, CH-1920 Martigny, Switzerland \\ ${ }^{2}$ École Polytechnique Fédérale de Lausanne, CH-1015 Lausanne, Switzerland \\ ${ }^{3}$ Electrical \& Computer Engineering, University of California, Santa Barbara, CA 93106, USA \\ ${ }^{4}$ Institute of Anatomy, University of Bern, CH-3000 Bern, Switzerland
}

\begin{abstract}
Microscopy of the beating heart in embryos provides key insights for the study of its development. However, achieving a sufficiently high framerate is difficult with conventional cameras. Here, we present a method to reconstruct an image sequence covering one heartbeat from images acquired over multiple cardiac cycles, with each image triggered at an arbitrary time, by sorting them according to their similarity. We formulate this task as a traveling salesman problem for which efficient solutions are available. We characterized our approach by evaluating its accuracy on synthetically generated data and sub-sampled high-speed movies of the beating heart in zebrafish larvae. We found that reconstructions are reliable when each phase produces a distinct image and when there are no abrupt cardiac motions, which amounts to collecting at least 100 images in a typical microscopy imaging scenario. We finally demonstrate that our method can be applied on data acquired with a fast confocal microscope, increasing its limited frame-rate by a factor 8 .
\end{abstract}

Index Terms - Microscopy, cardiac imaging, zebrafish.

\section{INTRODUCTION}

Live imaging of developing organs in embryos is possible within animal models such as the zebrafish. Imaging the heart comes with additional constraints as it beats already before it is fully developed and the microscope magnifies fast motions, which requires high frame-rates. Line scanning confocal microscopy [1], spinning disk microscopes (in some cases with strobed illumination [2]), or light sheet microscopes [3, $4,5]$, allow for fast framerates but are still limited by datatransfer bandwidth. Several techniques have been proposed

This work is supported by a project funded jointly by the Agence nationale de la recherche (ANR) and the Swiss National Science Foundation (SNSF), Grant 310030E-164245 "liveheart: The cellular basis of cardiac development revealed by live imaging," and the Swiss National Science Foundation Grant 206021_164022 "Platform for Reproducible Acquisition, Processing, and Sharing of Dynamic, Multi-Modal Data."

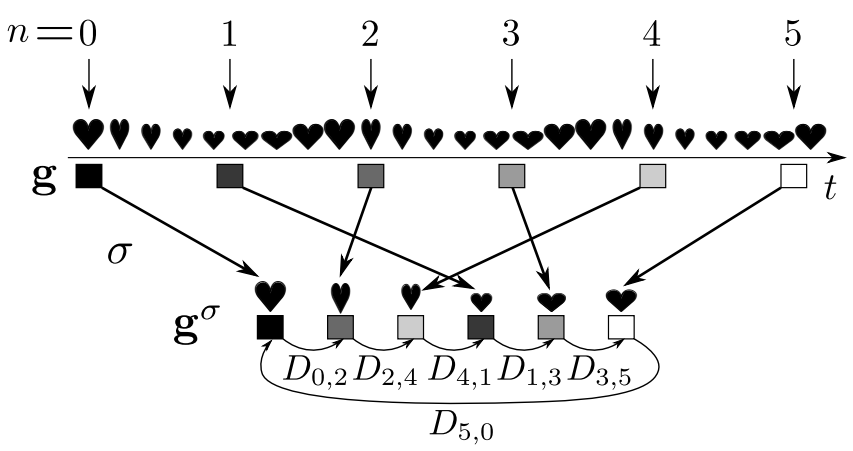

Fig. 1. Images of the beating heart are acquired at arbitrary phases of the cardiac cycle. We sort the acquired images such as to minimize the image-to-image distance $D_{k, \ell}$ between consecutive images, a task that we identify as a traveling salesman problem.

to build movies from image series acquired over multiple cycles of periodic processes when direct acquisition with a sufficient frame-rate was not possible. Zhang et al. [6] proposed to post-process MRI images of the beating heart acquired without gating in free-breathing subjects by learning the cardiopulmonary manifold formed by the images. This allowed them to sort the images over two axes, that they linked to the cardiac and the respiratory phases, respectively. Tralie et al. [7] used a tracking-free Eulerian approach for synthesizing slow motion videos from videos of multiple periods of a repetitive motion. In astronomy, techniques to assemble data acquired during irregularly-spaced observations to determined revolution periods $[8,9]$ inspired methods to determine the period in cardiac microscopy [1] and to virtually increase the framerate in cardiac optical coherence tomography [10]. However, the latter methods assume the underlying process is strictly periodic and that the acquisition time-stamps are known, which limits applicability and robustness.

In this paper, we propose a method that takes microscopy images of the beating heart, each triggered at an arbitrary time, and virtually increases the time resolution by sorting them to form a complete heartbeat (Fig. 1). The novel con- 
tributions in the paper are threefold. First, in Section 2 we formulate the task of sorting cardiac images as a traveling salesman problem for which efficient algorithms are available and whose output is a direct solution to the ordering problem (rather than a manifold projection). Second (in Section 3), we characterize our method on synthetic and experimental data. Third, we show an application to increase the framerate in an imaging modality, fluorescence microscopy, for which fast imaging is particularly challenging and which may therefore benefit from our method (Section 3). In Section 4, we discuss the performance and limitations of our method and conclude.

\section{METHODS}

\subsection{Imaging Model and Problem Statement}

We consider a time-varying two-dimensional image $f(x, y, t)$ with $(x, y)$ the spatial position and $t$ the time. The image intensity varies periodically with a period $T$, such that:

$$
f(x, y, t)=f(x, y, t+T) \quad \text { for all } t \in \mathbb{R} .
$$

We denote by $\mathbf{f}$ the image series covering one heartbeat:

$$
\mathbf{f}[k, \ell, n]=f\left(k \Delta_{x}, \ell \Delta_{y}, n \Delta_{t}\right),
$$

where $\Delta_{x}$ and $\Delta_{y}$ are the pixel width and height, respectively, $(k, \ell) \in \mathcal{K}=\{0, \ldots, K-1\} \times\{0, \ldots, L-1\}$ are the row and column index pairs, $n \in \mathcal{N}=\{0, \ldots, N-1\}$ denotes the time frame index, and $\Delta_{t}=\frac{T}{N}$ the time interval.

Given that $\Delta_{t}$ must be small (to achieve sufficient temporal resolution), it is typically not possible to acquire $\mathbf{f}$ directly. Instead, we consider the image series $\mathbf{g}[k, \ell, n],(k, \ell) \in \mathcal{K}$ :

$$
\mathbf{g}[k, \ell, n]=f\left(k \Delta_{x}, \ell \Delta_{y}, t_{n}\right),
$$

where $t_{n}, n \in \mathcal{N}$, denotes the times at which the frames were acquired. These times occur at arbitrarily-spaced times with: $t_{0}<\ldots<t_{n}<\ldots<t_{N-1}$, and $t_{n} \in \mathbb{R}$.

The image reconstruction problem we address is obtaining an estimate $\tilde{\mathbf{f}}$ of the uniformly sampled image sequence $\mathbf{f}$ given the measurements $g$, without knowledge of the underlying heartbeat period $T$ nor the starting times $t_{n}$.

\subsection{Virtual Frame-Rate Improvement via Sorting}

The key insight for reconstructing $\tilde{\mathbf{f}}$ is that the underlying signal $f$ is repeating and that, as we collect sufficient frames to build the data $\mathbf{g}$ (i.e. $N$ sufficiently large), we eventually capture the heart in all its poses, under the assumption that acquisitions occur at arbitrary times, unrelated to the period $T$. We further assume that the heart takes up poses that are distinct for each phase of the heartbeat and that only small changes are required to transform one image into the next. We propose to estimate $\tilde{\mathbf{f}}$ by sorting the frames $\mathbf{g}[:,:, n], n \in \mathcal{N}$.
We define the cardiac phase $\phi_{n}$ of the $n^{\text {th }}$ frame $\mathbf{g}[:,:, n]$ as the wrapping operation:

$$
\phi_{n}=\mathcal{W}_{T}\left(t_{n}\right)=t_{n}+m T, m \in \mathbb{Z} \text { s.t. } \phi_{n} \in[0, T) .
$$

The task of sorting the frames is equivalent to finding a permutation $\sigma: \mathcal{N} \rightarrow \mathcal{N}, m \mapsto n=\sigma(m)$ such that the phases $\phi_{\sigma(0)}, \ldots, \phi_{\sigma(N-1)}$ are in increasing order:

$$
\phi_{\sigma(0)} \leq \cdots \leq \phi_{\sigma(N-1)} .
$$

Given the sequence $\mathbf{g}$, we denote by $\mathbf{g}^{\sigma}$ a permuted frame sequence obtained by applying the permutation $\sigma$ to $\mathbf{g}$ :

$$
\mathbf{g}^{\sigma}[:,:, m]=\mathbf{g}[:,:, \sigma(m)], \quad m \in \mathcal{N} .
$$

We formulate the phase-ordering problem as a minimization task where we seek a permutation that minimizes the frameto-frame image difference between neighboring frames. We define the cost of a given candidate permutation $\sigma^{\prime}$ (with $\left.\sigma^{\prime}\left(t_{0}\right)=0\right)$ as:

$$
\begin{array}{r}
C\left(\mathbf{g}, \sigma^{\prime}\right)=\sum_{m=0}^{N-2} d\left(\mathbf{g}^{\sigma^{\prime}}[:,:, m], \mathbf{g}^{\sigma^{\prime}}[:,:, m+1]\right)+ \\
d\left(\mathbf{g}^{\sigma^{\prime}}[:,:, N-1], \mathbf{g}[:,:, 0]\right),
\end{array}
$$

where the frame-wise distance operator $d(\cdot, \cdot)$ between two $2 \mathrm{D}$ frames $\mathbf{x}$ and $\mathbf{y}$ is defined as:

$$
d(\mathbf{x}, \mathbf{y})=\sum_{k=0}^{K-1} \sum_{\ell=0}^{L-1}|\mathbf{x}[k, \ell]-\mathbf{y}[k, \ell]| .
$$

The permutations that lead to a minimal cost come in pairs (given that the image distance is symmetrical):

$$
\left\{\tilde{\sigma}^{T}, \tilde{\sigma}\right\}=\underset{\sigma^{\prime} \in S_{N-1}}{\arg \min } C\left(\mathbf{g}, \sigma^{\prime}\right)
$$

where $\tilde{\sigma}$ is the ordering that satisfies Eq. (5), and $\tilde{\sigma}^{T}$ the same permutation in reverse order.

We identify Problem (9) as an incarnation of the traveling salesman problem (TSP): the permutation we seek corresponds to finding a path that visits each image (and comes back to the starting image) while minimizing the distance traveled between adjacent images. Since there are $(N-1)$ ! possible frame permutations (the first frame is fixed) exploring all combinations would be prohibitively expensive. Instead, we use the TSP solver package Concorde [11], with the linear programming solver QSopt [12]. It takes an $N \times N$ (symmetrical) table $\mathbf{D}$, whose entries $D_{j, k}=d(\mathbf{g}[:,:, j], \mathbf{g}[:,:, k])$ contain the frame-wise distances between all frame pairs. The computational complexity of the Concorde solver is $\mathcal{O}\left(a \cdot b^{\sqrt{N}}\right)$, with $a=0.21$ and $b=1.24194[13,14]$. To build the table $\mathbf{D}$ efficiently and robustly, we spatially-average and downsample each frame $\mathrm{g}[:,:, n], n \in \mathcal{N}$ before computing the image distances. 


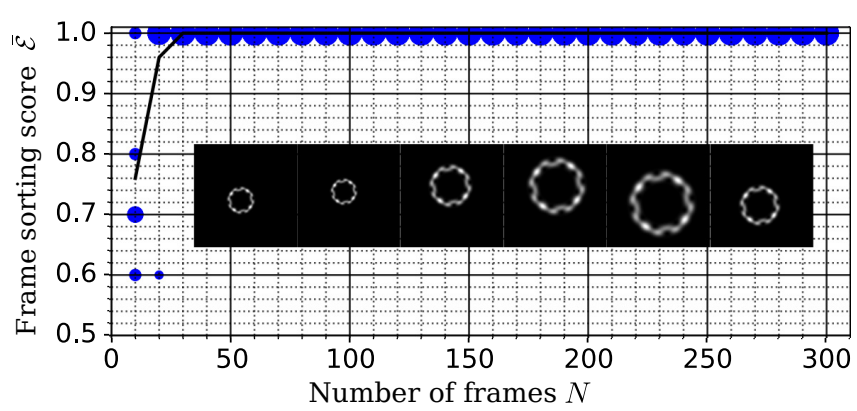

Fig. 2. Cardiac image sorting score as a function of the number of available images $N$. Frames were selected from a synthetic image series ( $K=L=256$ ) with phases drawn randomly from a uniform distribution (10 realizations per experiment). The sorting is near perfect for $N \geq 100$.

\section{RESULTS}

\subsection{Method Characterization on Synthetic Data}

To characterize the accuracy of the sorting process as a function of the number of available frames, we produced synthetic data of a ring contracting periodically and asymmetrically [1] over multiple periods. We selected $N \in\{10,20, \ldots, 300\}$ frames, whose temporal phases were drawn from a uniform distribution, and applied our sorting algorithm. To quantify the sorting accuracy, we defined the score:

$$
\begin{aligned}
\overline{\mathcal{E}} & =\left|\frac{1}{N} \sum_{n=0}^{N-1} \mathcal{E}[n]\right|, \text { with } \\
\mathcal{E}[n] & =\left\{\begin{aligned}
1 & \phi_{\tilde{\sigma}(n)}<\phi_{\tilde{\sigma}\left(\langle n+1\rangle_{N}\right)} \\
1 & \phi_{\tilde{\sigma}(n)}=\phi_{\max } \text { and } \phi_{\tilde{\sigma}\left(\langle n+1\rangle_{N}\right)}=\phi_{\min } \\
-1 & \text { otherwise, }
\end{aligned}\right.
\end{aligned}
$$

where $\phi_{\min }$ and $\phi_{\max }$ are the minimal and maximal ground truth phases, respectively and $\langle n\rangle_{N}=n \bmod N$. When correctly sorted, the phases should be in a strictly increasing or decreasing order, with a single jump between the minimal and maximal phases $\phi_{\min }$ and $\phi_{\max }$. We have $0 \leq \overline{\mathcal{E}} \leq 1$, with a low score expressing poor and a high score expressing correct sorting (the score does not penalize the direction). We observed that the sorting is unreliable below $N=30$ frames, then stabilizes toward the best score as $N$ increases. In additional experiments (not shown), we synthesized a motion that did not produce unique images for different phases (breaking an assumption for our method to perform correctly), which produced unpredictable sorting results (e.g. inverting segments in time between similar images).

\subsection{Validation on Experimental Measurements}

We bred wild type as well as transgenic $\mathrm{Tg}$ (myl7:membranemCherry) [15] zebrafish according to standard procedures [16]. The latter express red fluorescent protein in the muscle

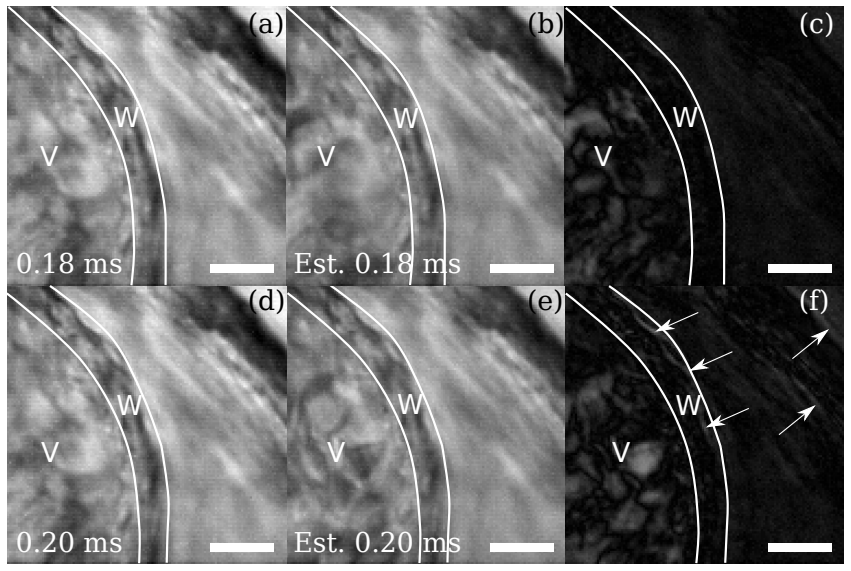

Fig. 3. Sorting evaluation on experimental data. (a),(d) Two frames from a 190 frame high-speed sequence of the beating zebrafish heart, acquired at $1000 \mathrm{fps}$ (ground truth). (b),(e) Corresponding frames obtained by sorting 190 randomly drawn from a 32,001-frame high-speed sequence. (c),(f) difference between ground truth and reconstruction. V: ventricle, W: heart wall. Arrows in (f) indicate areas of mismatch. Scalebar is $100 \mu \mathrm{m}$. See also Supplementary movie part 1 ([00:00-00:14]).

cells of the atrium. All procedures were authorized by the Institutional Animal Care and Use Committee at the University of California, Santa Barbara (wild-type) and the Cantonal Veterinary Office, Bern. Briefly, we grew the zebrafish embryos in E3 medium and added PTU (0.003\% 1-phenyl2-thiourea) to avoid pigmentation when the embryos reached an age of 24 hours post fertilization (hpf). At an age of 36 hpf, we removed the chorion surrounding the embryos with forceps and anesthetized the embryos with Tricaine at 0.08 $\mathrm{mg} / \mathrm{ml}, \mathrm{pH} 7$. We embedded the embryos with the ventral side down on a glass bottom dish in $1 \%$ low melting agarose.

\subsubsection{Validation on high-speed data of the heart}

We imaged the wild type heart at 55 hpf on a Leica DMR microscope equipped with a FASTCAM SA3 camera, using transmission, at a frame rate of 1,000 fps. We collected a 32,001-images dataset which covered about 76 heartbeats. We identified the first 380 frames (covering $0.38 \mathrm{~s}$ ) as a ground truth heartbeat, which we averaged pairwise to form a sequence of $N=190$ frames. Next, we randomly selected $N=190$ frames among the full dataset with indices drawn from a uniform distribution over the entire dataset, simulating a slow acquisition procedure. We next applied our sorting method and compared it with the 190 frames of the ground truth period (Fig. 3). The periodic parts of the ground truth and reconstructed images agree, demonstrating the correct ordering in experimental data. However, the red-blood cells, which are different from one heart-beat to the next differ. 


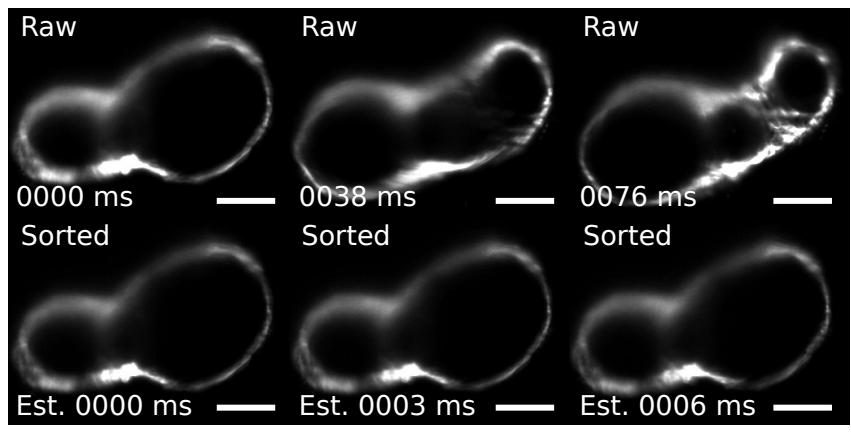

Fig. 4. Virtual framerate increase (202 fps) via sorting of 100 frames across about 8 heartbeats acquired at $26.3 \mathrm{fps}$ on a confocal microscope. Top row: three consecutive frames from raw sequence. Bottom row: three consecutive frames after virtual framerate increase. Scalebar is $50 \mu \mathrm{m}$. See also Supplementary movie part 2 ([00:15-00:40]).

\subsubsection{Virtual Framerate Increase in Fluorescence Microscopy}

To demonstrate the potential of our method for limited framerate modalities, we imaged the heart of the $36 \mathrm{hpf}$ old Tg(myl7:membranemCherry) zebrafish on a Zeiss LSM880 inverted confocal microscope with an LD C-Apochromat $40 \times / 1.1$ NA water immersion objective lens, in fluorescence, at $26.3 \mathrm{fps}$. We collected 100 frames. We applied our method to virtually increase the framerate by sorting all 100 frames into one single period (Fig. 4). Given the observed heartbeat covered 13 frames in the original dataset, the virtual frame achieved was $202 \mathrm{fps}$, a 7.7-fold increase. Note that only the framerate (not the temporal resolving power) is increased.

\section{DISCUSSION AND CONCLUSION}

Our sorting approach reaches a good accuracy when $N \geq$ 100. In particular, the standard deviation becomes smaller with an increasing $N$, with a mean around $99 \%$. Our method has two limitations. First, it requires that the sampling be unrelated to the cardiac period (to avoid stratified sampling) which is typically the case because the heart exhibits variations from one heartbeat to the next. Second, the signal must be asymmetric. For instance, imaging just a part of the cardiac heart wall or downsizing the signal too much can lead to a loss of asymmetry in the signal and failure to reconstruct the correct order. Artificially increasing the framerate of an image series is particularly promising for modalities that have too slow a framerate or whose writing bandwidth is insufficient or irregular.

\section{REFERENCES}

[1] M. Liebling, A. S. Forouhar, R. Wolleschensky, B. Zimmerman, R. Ankerhold, S. E. Fraser, M. Gharib, and M. E. Dickinson, "Rapid three-dimensional imaging and analysis of the beating embryonic heart reveals functional changes during development," Dev. Dynam., vol. 235, no. 11, pp. 2940-2948, Nov 2006.

[2] D. W. Staudt, J. Liu, K. S. Thorn, N. Stuurman, M. Liebling, and D. Y. R. Stainier, "High-resolution imaging of cardiomyocyte behavior reveals two distinct steps in ventricular trabeculation," Development, vol. 141, no. 3, pp. 585-593, 2014.

[3] J. Huisken and D. Y. R. Stainier, "Selective plane illumination microscopy techniques in developmental biology," Development, vol. 136, no. 12, pp. 1963-1975, Jun 2009.

[4] J. M. Taylor, J. M. Girkin, and G. D. Love, "High-resolution 3D optical microscopy inside the beating zebrafish heart using prospective optical gating," Biomed. Opt. Express, vol. 3, no. 12, pp. 3043-3053, Dec 2012.

[5] P. Mahou, J. Vermot, E. Beaurepaire, and W. Supatto, "Multicolor two-photon light-sheet microscopy," Nat Meth, vol. 11, no. 6, pp. 600-601, Jun 2014.

[6] Q. Zhang and R. Pless, "Segmenting Cardiopulmonary Images Using Manifold Learning with Level Sets," in Computer Vision for Biomedical Image Applications. Springer, Berlin, Heidelberg, Oct 2005, pp. 479-488.

[7] C. J. Tralie and M. Berger, "Topological eulerian synthesis of slow motion periodic videos," in 2018 25th IEEE International Conference on Image Processing (ICIP), Oct 2018, pp. 35733577.

[8] R. F. Stellingwerf, "Period determination using phase dispersion minimization," The Astrophysical Journal, vol. 224, pp. 953-960, Sep 1978.

[9] M. M. Dworetsky, "A period-finding method for sparse randomly spaced observations or "How long is a piece of string?"," Mon Not R Astron Soc, vol. 203, no. 4, pp. 917-924, Aug 1983.

[10] C. M. Happel, J. Thommes, L. Thrane, J. Mnner, T. Ortmaier, B. Heimann, and T. M. Yelbuz, "Rotationally acquired fourdimensional optical coherence tomography of embryonic chick hearts using retrospective gating on the common central Ascan," J Biomed Opt, vol. 16, no. 9, Sep 2011.

[11] W. Cook, "Concorde Home." [Online]. Available: http://www.math.uwaterloo.ca/tsp/concorde.html

[12] D. Applegate, W. Cook, S. Dash, and M. Mevenkamp, "QSopt Linear Programming Solver." [Online]. Available: http://www.math.uwaterloo.ca/ bico/qsopt/index.html

[13] D. L. Applegate, R. E. Bixby, V. Chvatal, and W. J. Cook, The Traveling Salesman Problem: A Computational Study (Princeton Series in Applied Mathematics). Princeton, NJ, USA: Princeton University Press, 2007.

[14] H. H. Hoos and T. Stützle, "On the empirical time complexity of finding optimal solutions vs proving optimality for Euclidean TSP instances," Optim. Lett., vol. 9, no. 6, pp. 12471254, Aug 2015.

[15] S. Rohr, C. Otten, and S. Abdelilah-Seyfried, "Asymmetric involution of the myocardial field drives heart tube formation in zebrafish," Circ. res., vol. 102, pp. e12-e19, Mar 2008.

[16] M. Westerfield, The Zebrafish Book : A Guide for the Laboratory Use of Zebrafish, 4th ed. Univ. of Oregon Press, Eugene, 2000. 